\title{
Le protocole prérédactionnel dans les manuscrits de L'Idiot de la famille
}

\section{Gilles Philippe}

\section{(2) OpenEdition \\ Journals}

Édition électronique

URL : http://journals.openedition.org/recherchestravaux/239

DOI : 10.4000/recherchestravaux.239

ISSN : 1969-6434

Éditeur

UGA Éditions/Université Grenoble Alpes

\section{Édition imprimée}

Date de publication : 15 octobre 2007

Pagination : 139-150

ISBN : 978-2-84310-111-3

ISSN : 0151-1874

\section{Référence électronique}

Gilles Philippe, "Le protocole prérédactionnel dans les manuscrits de L'Idiot de la famille », Recherches \& Travaux [En ligne], 71 | 2007, mis en ligne le 15 avril 2009, consulté le 08 septembre 2020. URL : http://journals.openedition.org/recherchestravaux/239; DOI : https://doi.org/10.4000/ recherchestravaux.239 


\section{Le protocole prérédactionnel dans les manuscrits de L'Idiot de la famille}

Les brouillons archivistiques, c'est-à-dire les notes prérédactionnelles que l'on prend pour stocker une information à développer plus tard, posent deux grands problèmes théoriques : celui de la spécificité de leur nature pragmatique (ressortissent-ils ou non au protocole communicationnel censé régir toute production discursive ?) et celui de la singularité de leurs formes linguistiques (trouve-t-on un appareil grammatical similaire dans d'autres productions écrites ?). Cette double entrée théorique permet d'opposer les notes sur Madame Bovary prises par Sartre en 1973 en vue du tome IV de L'Idiot de la famille à l'ensemble des brouillons des trois premiers tomes de l'ouvrage, rédigés de I968 à I97I et publiés en I97 I et I972. Les questions théoriques que l'on vient de poser sont cependant trop larges pour qu'on puisse leur trouver une réponse en se fondant sur un seul corpus, fût-il prototypique. Aussi, sans abandonner l'ambition d'une généralisation des analyses que l'on va proposer, va-t-on en réduire considérablement l'empan. On s'intéressera en effet successivement au statut génétique, pragmatique et grammatical des notes archivistiques de L'Idiot de la famille en mettant d'abord en regard les pratiques d'écriture et l'imaginaire langagier de Sartre. On verra alors quelles conclusions peuvent être tirées pour l'ensemble des protocoles génétiques sartriens, qu'ils soient prérédactionnels ou rédactionnels.

\section{Le statut génétique du protocole archivistique}

Pour comprendre le type d'acte génétique effectué par Sartre dans les notes prises pour le tome IV de L'Idiot de la famille, il faut se représenter la spécificité 
de ces quelques dizaines de pages, par rapport aux milliers de feuillets noircis pour les tomes précédents. Certes, la rédaction de l'ouvrage ne commença pas en i 968, lorsque Sartre entama l'écriture de L'Idiot tel que nous le connaissons, mais une douzaine d'années plus tôt, avec la mise en chantier d'une première étude sur Flaubert ${ }^{1}$. Des manuscrits de celle-ci (I954-1955), aujourd'hui en mains privées, nous savons hélas peu de choses : il s'agirait d'une quinzaine de blocs de "papier Sartre ${ }^{2}$ ", dont une partie au moins pourrait être une mise au net (rédaction continue, sans altérations et sur l'ensemble du feuillet, contrairement à la pratique habituelle de Sartre pour les rédactions non définitives). Des manuscrits de la deuxième version du Flaubert (1964-1965), sans doute dispersés de façon plus aléatoire et désormais probablement répartis entre de multiples collections privées, nous ne savons rien. La Bibliothèque nationale de France détient, en revanche, une partie très importante des manuscrits de la dernière version du texte, celle que Sartre fit paraittre dans la «Bibliothèque de philosophie » des éditions Gallimard ${ }^{3}$.

Cet ensemble de près de 6000 feuillets n'a pas encore fait l'objet d'un foliotage définitif, et de nombreux passages présentent des numérotations concurrentes (sans doute de la main de Simone de Beauvoir, Michèle Vian ou Arlette Elkaïm-Sartre). Il ne constitue pas, par ailleurs, l'intégralité des avanttextes de l'ultime version du Flaubert, puisque des pages autographes de l'œuvre passent encore régulièrement en vente chez les libraires. Il est en effet possible qu'un des familiers de Sartre ait obtenu de l'auteur d'autres sections manuscrites du texte, même si les lots de brouillons entrés à la Bibliothèque nationale de France dans les années i 980, se complètent parfaitement, ce qui laisse penser que l'institution conserve désormais l'essentiel des avant-textes de la version publiée de L'Idiot de la famille.

Ces pièces sont parvenues à la Bibliothèque nationale en trois vagues. En I 982 , tout d'abord, la fille adoptive de Sartre, Arlette Elkaïm-Sartre, a fait don d'une mise au net et d'une dactylographie de la préface du livre (identiques à l'état publié), ainsi que d'un lot de 898 feuillets autographes (à l'exception de quelques dactylogrammes), correspondant aux 660 pages des livres 2 et 3 de la deuxième partie. En 1985 , ensuite, la Bibliothèque a acquis l'essentiel des

I. Pour l'histoire du texte et quelques informations complémentaires sur les manuscrits disponibles, nous renvoyons aux Repères chronologiques publiés à la fin de ce volume.

2. On appelle " papier Sartre » le support le plus usuellement utilisé par l'auteur après la Guerre (blocs de feuillets à carreaux verticaux, de format 2I x $27 \mathrm{~cm}$, souvent de la marque Diane). Sartre écrit généralement au seul recto.

3. Les remarques qui suivent doivent énormément à Mauricette Berne, qui a constitué le fonds Sartre du département des manuscrits de la Bibliothèque nationale de France. Qu'elle en soit très vivement remerciée. Toute ma gratitude va aussi à Anne Mary, qui gère désormais ce fonds et m'a aidé à accéder aux documents. 
manuscrits donnés par Sartre à Michèle Vian. Parmi ceux-ci figure un lot d'environ 5000 feuillets d'avant-textes de L'Idiot. Il s'agit clairement d'une version de travail, qui mêle, à l'ouverture, feuillets autographes sur papier Sartre et feuillets dactylographiés (pour environ un quart de l'ensemble). Le « lot Vian » correspond à la première partie et au livre I de la deuxième partie de L'Idiot (soit environ I Ioo pages publiées). Même en tenant compte de la présence des dactylogrammes et des incertitudes dues aux pertes de feuillets, on voit que le travail sur la première partie du livre fut bien plus important que pour la suite, qui a été rédigée par Sartre de façon presque continue. Un feuillet dactylographié de couleur à l'entrée du manuscrit prévoit, semble-t-il, que l'ouvrage porte en exergue la citation de la lettre d'octobre i 864 qui sera plus tard glosée dans la préface qui relie L'Idiot de la famille à Questions de méthode, préface dont la nécessité semble s'être imposée assez tardivement à l'auteur : «C'est à force de travail que j'arrive à faire taire ma mélancolie native. Mais le vieux fond reparait souvent, le vieux fond que personne ne connait, la plaie profonde toujours cachée. » En I989, enfin, la dation de la fille adoptive de Simone de Beauvoir, Sylvie Le Bon-de Beauvoir, a fait entrer à la Bibliothèque nationale, un lot de 1900 feuillets de L'Idiot; ceux-ci portent une numérotation continue de I à 208 I, mais il manque une séquence de I $8 \mathrm{I}$ feuillets. Ce manuscrit correspond à la troisième partie du texte (fin du tome II), et aux deux livres qui forment le tome III (soit, environ, I oo० pages publiées). Plusieurs centaines de feuillets de ce lot présentent un développement non retenu par Sartre et toujours inédit. Les documents disponibles confirment donc les témoignages des proches de Sartre : la rédaction du tome III de L'Idiot s'est faite de façon continue, d'une traite, avec aisance et sans brouillonage 4 .

Le changement de protocole génétique, lorsque, en 1973, Sartre entreprend le tome IV de son livre, est donc spectaculaire. Cette fois, l'auteur consigne dans deux cahiers une longue série de notes : le plus souvent de simples relevés documentaires, parfois des ébauches rédigées'. Nous ne connaissons que le premier de ces cahiers, légué en 2005 par A. ElkaïmSartre à la Bibliothèque nationale de France. Les notes sur Madame Bovary diffèrent donc matériellement des autres brouillons connus de L'Idiot de la famille. Le support, tout d'abord, est exceptionnel chez le dernier Sartre : un

4. Voir aussi ce que dit Sartre dans l'entretien donné au Monde le I 4 mai I 97 I : «Le livre a été écrit au fil de la plume» (Situation, X, Gallimard, 1976, p. 93).

5. Ces cahiers ont été transcrits et annotés par A. Elkaïm-Sartre en annexe du tome III de la réédition de L'Idiot de la famille ( 1988 ). Le cahier de la BNF (désormais «Cahier 2005 》) correspond aux pages 664-765 du texte paru en I988, soit aux deux premiers tiers; les listes brutes de références et de citations ayant été écartées par l'éditrice, on devine aisément la densité des pages du manuscrit-source. 
cahier cartonné de très grand format ${ }^{6}$, et non des blocs de feuillets détachables. Chaque page est, par ailleurs, utilisée dans sa totalité, et non sur son seul tiers ou sa seule moitié supérieure, comme Sartre aime à le faire (c'est le cas pour une bonne partie des lots I982, 1985 et I 989), afin de ne pas avoir à reprendre tout le feuillet, en cas de remords de rédaction. Enfin, l'écriture du cahier est plus serrée, moins lisible, manifestement plus rapide que ce qui s'observe dans le reste des manuscrits de l'ouvrage. Si de très nombreux mots sont soulignés, l'ensemble du cahier ne présente aucune altération, alors que dans les brouillons rédactionnels de Sartre, on observe fréquemment des biffures de plusieurs lignes, sans doute opérées à la relecture.

Avant toute considération pragmatique ou linguistique, ce simple constat codicologique oblige à remarquer que les premiers avant-textes du tome IV de L'Idiot de la famille suivent une démarche génétique tout à fait différente des trois premiers. On ne saurait cependant opposer sur cette seule base le Cahier 2005 aux lots précédents, et surtout en conclure que le protocole avant-textuel du tome IV permet à lui seul d'établir une différence de nature entre le dernier et les trois premiers tomes de L'Idiot. Il est en effet possible que nous ayons simplement affaire à des stades génétiques différents : le Cahier 2005 relèverait de la prise de notes documentaire (nous y trouvons de nombreuses listes ${ }^{7}$ ) ou prévisionnelle (nous y trouvons de nombreux plans), tandis que les trois autres lots correspondraient à un stade génétique ultérieur, proprement rédactionnel. Cependant, bien que les manuscrits du dernier Sartre aient probablement tous été conservés par ses proches (même s'ils ont pu être dispersés lors de ventes successives), aucun des milliers de feuillets connus des brouillons des trois premiers tomes de L'Idiot ne présente une telle prise de notes; il semble donc que, même si le matériel historique et biographique brassé par Sartre y est trop considérable pour n'avoir fait l'objet d'aucune mise à l'écrit avant le stade rédactionnel, cette prise de notes ait dû être bien limitée. On dira alors que l'objet si particulier du tome IV de L'Idiot, une étude textuelle positive, appelle un travail de repérage préalable que ne saurait requérir le « roman vrai» des tomes I à III, qui ne garantit jamais des contenus largement soumis à la fictionalisation. Or, cette donnée doit aussi probablement être

6. Il s'agit très exactement d'un in- $4^{\circ}$ « raisin », fabriqué dans l'Isère par la manufacture Le Dauphin (Voiron). Il a été acquis à la Papeterie du Dôme, ı 8 bd du Montparnasse, pour I I francs 50. Les 99 pages à petits carreaux sont rédigées au recto seul (à l'exception de quelques mots ou quelques lignes au verso des pages 4I, 43, 45, 49, 6I, 73 et 89), à l'encre bleue ; le foliotage au stylo-bille est allographe.

7. Les pages i6-36 du cahier sont ainsi presque exclusivement occupées par une liste de numéros de pages (du roman ou de la correspondance), simplement glosés d'un ou de quelques mots. 
écartée : la prise ou non de notes prérédactionnelles ne coïncide pas, chez Sartre, à une quelconque opposition générique. Les textes pour lesquels nous conservons des notes de ce type relèvent de tous les genres, fictionnels ou essayistiques, de la même façon que toutes sortes de textes, littéraires ou techniques, furent écrits « au fil de la plume »-selon l'expression employée par Sartre pour caractériser la rédaction des trois premiers tomes du Flaubert. La seule distinction pertinente en cette matière est celle qui oppose les textes publiés aux textes inachevés : le passage par la prise de notes prérédactionnelle témoigne - on ne saurait s'en étonner - d'une résistance dans la gestation du texte, résistance qui rend souvent prévisible son inachèvement. C'est ce qui se vérifie à nouveau sur les brouillons de L'Idiot de la famille, et cette évidence peut servir de point de départ.

\section{Le statut pragmatique du protocole archivistique}

Avant d'en venir aux formes que revêt cette production linguistique si spécifique qu'est la note prérédactionnelle telle qu'elle apparait dans le Cahier 2005, il convient de réfléchir sur le statut pragmatique particulier de la prise de notes en général, mais aussi d'envisager la question du protocole archivistique dans la seule perspective du rapport de Sartre au langage. On peut en effet mettre en regard le Cahier 2005 et ces lignes de i 948 :

Sans doute [le langage] peut-il fixer les résultats de l'intuition, mais en ce cas quelques mots jetés à la hâte sur le papier suffiront : l'auteur s'y reconnaîtra toujours assez. Si les mots sont assemblés en phrases avec un souci de clarté, il faut qu'une décision étrangère à l'intuition, au langage même, soit intervenue : la décision de livrer à d'autres les résultats obtenus ${ }^{8}$.

Ces lignes sont en effet les seules du corpus sartrien où l'auteur évoque explicitement la prise de notes. Or, on le voit, celle-ci n'est pas conçue comme une production discursive marginale, mais, tout au contraire, comme celle où l'activité langagière est réalisée dans son état le plus pur, c'est-à-dire sans le parasitage des exigences discursives communicationnelles. La conception sartrienne du langage qui s'exprime ici est bien sûr redevable de l'héritage bergsonien de la philosophie française, mais elle catalyse aussi involontairement certaines thématiques qui apparaissent après la Guerre et dont la variante française du chomskysme fournira bientôt les formulations les plus nettes. On le sait, pour Chomsky, la vocation première du langage n'est pas la communication, mais l'assistance à la cognition; on le sait aussi, pour Bergson, le

8. Situations II (Qu'est-ce que la littérature ?), I 948, Gallimard, «Folio », I98 5, p. 28. 
langage ne joue qu'un rôle mineur dans la cognition : la mise en mots est l'ultime étape, extérieure et périlleuse, du processus intellectif. Vu sous cet angle, le compromis qui apparaît chez Sartre ne semble conserver que le pessimisme des deux positions. La note, puisque c'est bien d'elle qu'il s'agit dans ces lignes, joue essentiellement un rôle de fixation de l'intuition - c'est-à-dire moins de clarification que de mémorisation externe ; le langage reste pour Sartre, comme pour Bergson, extérieur au processus intuitionnel proprement dit. Or, si l'intuition se dégrade dans son expression langagière, l'expression langagière se dégrade à son tour, nous dit Sartre, quand elle est soumise aux impératifs communicationnels.

On dira que ces lignes de Situations II sont trop isolées pour servir de point de départ. Il n'en est rien. En effet, s'il n'y a pas de philosophie du langage stable chez Sartre (l'évolution est considérable entre ce qui est dit dans chacune de ses deux sommes philosophiques, celle de 1943 et celle de 1960), l'imaginaire de la langue reste étonnamment inchangé dans l'ensemble du corpus sartrien', et les lignes que nous commentons en sont particulièrement représentatives. On peut d'ailleurs les ramener à deux grandes thématiques, sans cesse reformulées d'un bout à l'autre de l'œuvre. La première est celle de la «nomination », si l'on retient le terme qui apparait dans Les Mots ${ }^{10}$ : appliquer un mot à un objet, concret ou abstrait, le constitue en notion cognitivement maniable. Cette mise en avant du processus nominatif chez Sartre à deux conséquences, nettes dans les lignes de 1948 que nous citions : d'une part, le mot, et non la phrase, reste, pour lui, l'unité langagière première ; d'autre part, le processus prédicatif n'est pas le fondement de l'énoncé. Peu de temps avant de reprendre la rédaction de L'Idiot de la famille, Sartre insistait ainsi à nouveau sur le fait que, dans son rapport personnel au langage, la fonction de "thématisation » (équivalent linguistique de l'acte de nomination, c'est-à-dire cristallisation en notion d'un contenu intuitionnel diffus) primait sur la fonction de communication : « Le langage [...] est d'abord pour moi un objet qui m'enveloppe et dans lequel je peux prendre des choses, ensuite seulement je découvre sa fonction de communication ${ }^{11}$. »

La seconde thématique est consécutive de la première : c'est celle de l'«aliénation ». Digne héritier de Stendhal, Sartre pense en effet que «les mots

9. Voir Gilles Philippe, «L'analyse sartrienne de la phrase », Le Gré des langues, n I 2, I997, p. I $30-147$.

Io. Les Mots, Gallimard, I963, p. I I7. Cette thématique, déjà déterminante dans le texte sur Ponge de 1944 ("L'homme et les choses », repris dans Situations [I], Gallimard, I 947), hante encore Sartre lorsqu'il rédige L'Idiot de la famille: «J'ai toujours pensé que m'approprier la table, c'était trouver le mot sur la table » ("L'écrivain et sa langue », entretien avec P. Verstraeten, I965, repris dans Situations $I X$, Gallimard, 1972, p. 41).

I I. Ibid., p. 40. 
volent ma pensée » : d'une part parce que l'intuition individuelle s'exprime mal dans des expressions conventionnelles qui ne sauraient coïncider exactement avec elle, d'autre part parce que l'introduction de la moindre visée communicationnelle fait peser des contraintes sur la formulation. La transmission d'un contenu informationnel exact et original ne serait en effet possible qu'en prenant en considération l'horizon interprétatif du destinataire, afin de déjouer ou neutraliser ses attentes (c'est que Sartre explique dès L'Étre et le Néant: si je veux dire $\mathrm{x}$ et que je formule « $\mathrm{x}$ », l'horizon interprétatif du destinataire fait que celui-ci comprend x'; pour faire comprendre $x$, j'adopterai donc une formulation " $\mathrm{x}$ " ", qui tienne compte de ce tamis interprétatif et aboutisse chez le destinataire à une interprétation en $\mathrm{x}$ ). On ne s'étonnera donc pas si, quelques lignes avant celles que nous considérons, Sartre écrivait qu'« il est vrai que la parole [est] une trahison et que la communication [est] impossible ${ }^{12}$. » Que le langage serve d'abord à nommer ou à archiver le réel pour soi signifie donc, selon Sartre, que tout usage communicationnel asservit le langage à un emploi qui n'est pas d'abord le sien : le transfert d'informations vers un tiers. À ce parasitage de l'acte nominatif par l'acte communicatif n'échappent, pour Sartre, que deux productions langagières : la première complètement, c'est la note d'archive, comme nous l'avons vu ; la seconde partiellement, c'est la littérature qui «suppose la non-communication, parce que quand on dit que les écrivains écrivent pour autrui, ce n'est vrai qu'à la longue, ce n'est pas vrai originellement ${ }^{13}$. »

Si étonnant que cela puisse sembler, la note prérédactionnelle archivistique (du type de celles que l'on trouve dans les manuscrits du tome IV de L'Idiot, mais bien rarement dans ceux des tomes précédents) apparaît donc pour Sartre comme le prototype même de l'activité langagière, puisqu'elle est la seule qui n'ait pas à prendre en considération un destinataire et échappe donc à la volonté, «extérieure [...] au langage lui-même», de communiquer. Or, une telle position (dont on doit prendre acte comme un élément de l'imaginaire langagier sartrien, sans chercher à la discuter) va moins de soi qu'on pourrait le

I 2. Situations II, éd. citée, p. 47 .

I 3. «L'écrivain et sa langue », op. cit., p. 43. Voir aussi plus loin : «Quand j'écris ce qu'on appelle de la prose littéraire il y a toujours cet aspect-là, sinon ce ne serait pas la peine d'écrire dans cette langue; par contre, ce qui est le plus difficile pour la communication philosophique c'est qu'il s'agit de pure communication. » (p. 45). Pour Sartre, l'énoncé littéraire ne transfère pas d'informations : il «signifie », c'est-à-dire qu'il représente. Le lecteur « assiste » à la signification, sans en être le destinataire au sens strict : «Flaubert écrit et pense que nous ne pouvons pas communiquer, ce qui l'amène à créer un ensemble de significations qui doivent être ellesmêmes l'objet littéraire » (p. 49). Sur ce point, comme sur tant d'autres, la sensibilité au langage de Sartre coïncide assez étroitement avec celle de son écrivain préféré, Stendhal. Voir G. Philippe, «Stylistique et pragmatique du style», Ph. Berthier et É. Bordas dir., Stendhal et le style, Presses de la Sorbonne Nouvelle, 2005, p. I99-208. 
penser, dans sa conclusion bien sûr (la notation archivistique constitue le prototype de l'activité langagière), mais même et surtout dans son préalable (la notation archivistique est foncièrement non-communicationnelle). Celui-ci est en effet contesté par ceux-là même qui devraient le reconnaître (Derrida put ainsi affirmer que, pour Husserl, « le fait d'inscrire une pensée était toujours de l'ordre de la communication, éventuellement entre lui et lui ${ }^{14} \gg$ ).

On se trouve certes ici face à l'une de ces interrogations qui appellent cette régression à l'infini qui explique que certains problèmes théoriques soient condamnés à demeurer sans solution : pourquoi émettrait-on des énoncés dans des situations qui ne valideraient pas la dynamique communicationnelle qui les fonde ? La solution proposée par Sartre semble à cet égard assez satisfaisante ; elle assigne à la note d'archive la fonction d'un stimulus différé : je parie qu'en relisant mes notes, je pourrai reconstruire mon trajet cognitif original, et les notes sont prises de telle sorte que cette reconstruction soit aisée. La note n'est donc pas, à proprement parler, un message de soi à soi ou un cas spécifique de communication : elle ne me constitue pas en un «destinataire » de façon comparable à ce qui se passe dans un transfert d'informations d'un locuteur à un allocutaire. C'est d'ailleurs ce qui se vérifie, lorsqu'on s'attarde sur les formes langagières propres aux productions archivistiques.

\section{Le statut grammatical du protocole archivistique}

Si la position de Sartre sur la note d'archive semble pertinente, ce n'est pas tant à cause d'options théoriques indémontrables (la visée communicationnelle est « étrangère » ou non à la langue même, pour reprendre l'image de Sartre), qu'au titre des évidences linguistiques. Pour comprendre pourquoi la forme des notes prises pour le quatrième tome de L'Idiot de la famille diffère tant de celle des brouillons des trois premiers, on peut en effet prendre appui sur un postulat élémentaire : toute modification du régime pragmatique d'un discours a des conséquences sur sa forme grammaticale; réciproquement, la forme grammaticale d'un texte permet d'émettre des hypothèses sur sa spécificité pragmatique. En cela, les notes du Cahier 2005 ne témoignent pas d'un stade génétique différent des autres avant-textes du Flaubert, ou d'un travail en vue d'un type de texte différent, mais bien d'un acte discursif d'une autre nature.

I 4. Jacques Derrida, « Archive et brouillon (entretien)», M. Contat et D. Ferrer éd., Pourquoi la critique génétique?, CNRS Éditions, I998, p. I95. On pourra lire dans ce texte la réponse complexe que Derrida oppose à l'affirmation de Michel Contat : « les brouillons sont des textes sans destinataires » (p. I94-195). 
Rappelons-le, l’intentionnalité pragmatique (le statut communicationnel d'une production verbale) se vérifie sur trois données linguistiques, normalement toujours requises dans une production discursive standard : l'acte de prédication comme fondement de l'énoncé ; la distribution de l'information sur plusieurs plans; la textualisation des prédications ${ }^{15}$. On peut envisager sous cet angle le premier feuillet des notes prises par Sartre en vue du tome IV de L'Idiot $^{16}$ :

Un principe de toute sa psychologie : les atmosphères déterminent (ni faux ni vrai)

Le bal (besoin de richesse et d'élégance) la détermine à aller à Yonville

Pommier 52 : un peu putain

A Yonville : entrée de Bournisien : théâtrale

Lire la Corr sur Saint Antoine (peu de choses. En parle plus tard (au passé)

Le temps : un intermédiaire entre passé, présent et avenir : le fréquentatif

(imparfait), temps de l'éternel retour — éternité. S'oppose à l'événement

Cf Yonville : il y a l'événement — la passion d'Emma et sa mort

et puis le fréquentatif (qui demeure avec d'autres individus — les servantes -

pour faire les mêmes gestes)

Le temps : l'action disparaitt (cahier I 2 ) reste l'ennui (le vrai présent).

Psychologiquement : la maturation. Emma se donne à Léon quand elle est «mûre».

Mme B, roman de l'échec et de la fatalité. Pas de causes (Bouilhet les supprime? Parce qu'elles sont fausses ou parce que le lecteur comprendra?) Des maturations. Surtout le fond des personnages inconnu à F. « ou peut-être... sans doute...»

Les 2 manières d'aimer: Don Juan. Reprises dans Mme Bovary : Rodolphe Léon. Évidemment Charles a la première.

Hystérie de F. prêtée à Mme Bovary.

Le langage trahit les sentiments. Non de Charles qui ne parle pas ou

dit des banalités (mais alors il est insuffisant à vivre) ni de Rodolphe (qui est tout négatif)

Emma et Mazza : d’abord les sens non développés puis ils se développent (surtout Léon) et deviennent contraignants, l'emportent, créent la mort.

I 5. L'apparition des données strictement communicationnelles — l'affichage d'un statut illocutoire ou l'explicitation de la scénographie énonciative — sont en revanche non obligatoires. Leur absence n'est donc pas discriminante. On notera des infinitifs dits « d'auto-injonction» dans les lignes du Cahier 2005 que nous transcrivons ici («Lire $[\ldots]$ ajouter $[\ldots]$ ); leur statut illocutoire et la manière dont ils convoquent une scène communicationnelle mériteraient une étude plus longue, qui nous éloignerait de notre problématique première.

I6. Nous donnons ici la transcription diplomatique de la première page du Cahier 2005 de la BNF (il n'est cependant pas certain que le cahier ait conservé tous ses feuillets). Les deux dernières lignes, en pied de page, d'une encre qu'on ne retrouvera qu'à la page 48, semblent avoir été ajoutées plus tard. Une édition pour la lecture courante en a été proposée par A. ElkaïmSartre en annexe du tome III, dans la nouvelle édition de L'Idiot de la famille (Gallimard, I988, p. 665-666). 
Insincérité du langage (cahier XIII) : ajouter le langage muet, sans paroles (ils se regardent dans les yeux) qui est sincère.

La définition de l'Art : la pensée rendue par la forme (Souv. der nière page) exprimée ici

Pommier 170 On remit à causer... Inspire la correction on se remit à (Pléi. 348) qui veut dire le contraire. Le rêve sur les mots.

Autre exemple: si l'Église l'interdit, c'est qu'elle a ses raisons (Pom.). Pl : c'est qu'elle a raison.

On a dit que l'on tenterait principalement d'analyser le protocole archivistique de L'Idiot de la famille sur le fond de la pratique et de l'imaginaire sartriens de la langue. De ce point de vue, le premier des trois critères énumérés plus haut est de loin le plus intéressant. Sans revenir sur la disqualification de la « phrase » comme unité langagière première pour Sartre, on voit que la structure la mieux représentée ici est celle qui, à l'alinéa, semble isoler une donnée thématique ou scénique (de nature grammaticale quelconque), qui sera suivie par une donnée prédicative : «Psychologiquement : la maturation », «La définition de l'Art : la pensée rendue par la forme », etc. Rien en cela de très étonnant : l'effacement de l'appareil syntaxique en général, ou des formes sémantiquement ténues (le verbe être en copule attributive, par exemple), est requis par le principe d'économie linguistique propre à la pratique archivistique. Mais on voit bien que la spectaculaire surreprésentation de la structure $x: y$ et surtout son extrême plasticité (on a toutes sortes de composantes à droite et à gauche des deux points) ne correspond pas tant, dans l'imaginaire de Sartre, à une construction prédicative unique avec effacement du verbe, qu'à deux mouvements prédicatifs : le premier délimitant un contenu thématique, le second procédant à une assignation attributive ou processuelle. Cet ordre, qui correspond pour Sartre au fonctionnel intuitionnel standard ${ }^{17}$, serait celui-là même de son style spontané :

Guille se moquait de moi en ces termes : «On disait de Jules Renard qu'il finirait pas écrire : la poule pond. Mais toi, tu écrirais : il y a la poule et elle pond. „Cela est vrai : par le «Il y a », je séparerais avec plaisir la poule du reste du monde, j'en ferais un petit absolu tranché et immobile et je lui attribuerais la ponte comme une propriété, un attribut. Il y a quelque chose de transitif dans «La poule pond», qui me déplaît fortement, qui fait s'évanouir la «substance » poule en une pluralité de rapports et d'actes ${ }^{18}$.

17. On trouve d'ailleurs de nombreuses variations sur cette structure dans la représentation romanesque du discours intérieur chez Sartre. Voir G. Philippe, Le Discours en soi, Champion, I 997 , p. 389-390.

i 8. Carnets de la drôle de guerre [1939], Gallimard, I995, p. 282. L'anecdote et l'analyse seront reprises pour commenter le style de Camus en I 943 ("Explication de L'Étranger», Situations [I], éd. citée, p. I Io-I I I). 
Dans le premier feuillet du Cahier 2005, parce que nous avons affaire à des notations prérédactionnelles, le rapport logique entretenu par les unités en aval et en amont des deux points (dont l'omniprésence chez Sartre est un stylème d'autant plus fort que l'auteur ne leur assigne jamais de valeur précise) est encore plus souple que le simple décumul du système thème-prédicat en deux prédications, l'une existentielle (ily a x...), l'autre attributive ou processuelle $(x+v e r b e)$. La première «nomination » (il ne s'agit pas forcément de substantifs) fait apparaître un contenu thématique, référentiel ou non, la seconde un autre élément, qui entre avec le premier dans un rapport prédicatif flou, que la structure syntaxique ne précise pas.

On a vu que, pour Sartre, la grammaticalisation des relations logiques (le passage de la juxtaposition des syntagmes à leur organisation phrastique) correspondait au passage de la fonction fondamentale (cognitive) du langage à sa fonction dérivée (communicationnelle). Mais cette absence d'un tiers-destinataire s'observe ici bien au delà de la négociation des structures prédicatives, c'est-à-dire dans les procédures de textualisation et de hiérarchisation des prédications. On le comprend mieux si l'on garde en mémoire le fait que la prose d'idées de Sartre est spectaculairement ultracohésive (elle soude tout par l'adverbe ou l'anaphore) et hyperhypotaxique (elle articule les contenus propositionnels en des chaînes rigoureuses de subordonnées) ${ }^{19}$. Ce qui doit retenir notre attention ici, c'est donc moins la forme linguistique même des notes archivistiques du Cahier 2005, somme toute fort banale, que ce que révèle le contraste entre les pratiques prérédactionnelles et les pratiques rédactionnelles sartriennes. Nous l'avons dit, l'obsession de Sartre lorsqu'il rédige de la prose non littéraire, celle qui relève pour lui de la « communication pure », c'est la possibilité que l'interprétation du texte soit faussée par les inférences indues du lecteur. L'ensemble des caractéristiques formelles observables dans les avant-textes des trois premiers tomes de L'Idiot (et, partant, dans le texte publié) obéissent donc pour Sartre à la contrainte de transformer des contenus intuitifs en informations transférables à un destinataire, selon un tissage si étroit que le lecteur ne puisse sortir du raisonnement. $\mathrm{Si}$ l'on continue à observer les formes dans la seule optique du rapport imaginaire de Sartre à la langue, on voit alors comment une opposition théorique rudimentaire - celle qui oppose la fonction cognitive à la fonction communicative de langage - s'incarne dans des oppositions formelles à peine moins rudimentaires : recours fréquent à l'alinéa, asyndète et hyperparataxe, hiérarchisation de l'information par parenthésage, accumulation progressive de

I 9. Sur ces points, nous devons renvoyer aux analyses proposées dans G. Philippe, «La nostalgie du style ? Réflexions sur l'écriture philosophique de Sartre », Rue Descartes, n 47, 2005, p. $45-53$. 
données, chaînes référentielles lâches... dans le feuillet d'ouverture du Cahier 2005 ; très longs paragraphes, abondance de connecteurs, hiérarchisation hyperhypotaxique des informations, déplacement en tête de phrase de nombreuses données, anaphorisation systématique... dans les avant-textes et le texte publié des premiers tomes de L'Idiot.

Il serait imprudent de prétendre aboutir à des conclusions d'ordre général sur le statut pragmatique et la spécificité linguistique de la note d'archive à partir des seuls constats que nous venons d'opérer sur le Cahier 2005 de L'Idiot de la famille. Mais on voit que les problématiques ouvertes dans la confrontation entre le matériel génétique et l'imaginaire langagier de Sartre permettent de mieux comprendre ses pratiques rédactionnelles en général. Il convient en effet de rouvrir le programme proposé à l'analyse littéraire en I 925 par le critique britannique I. A. Richards : selon les Principles of Literary Criticism, la première question que l'on doit résoudre dans l'analyse des textes, c'est celle de l'acte même que l'auteur croit être en train d'accomplir; et cette question doit se poser - dans un premier temps - indépendamment de toute considération sur l'effectivité de l'acte discursif accompli. Or, dans l'imaginaire de Sartre, la pratique «pure» du langage, celle qui correspondrait à son style spontané, c'est bien celle de la notation archivistique sans visée communicationnelle aucune. L'ensemble des procédures grammaticales ou textuelles qui font l'originalité si évidente de la prose d'idées sartrienne ${ }^{20}$ et constituent pour le lecteur son « style » propre sont donc pour Sartre, à l'inverse, de simples concessions à la nécessité de transmettre efficacement des informations à un tiers. Loin de les percevoir comme «personnelles », il les associe à une contrainte de nature fondamentalement rhétorique, imposée du dehors. Cette dichotomie très forte, qui correspond chez Sartre à une opposition simple entre cognition et communication, mais aussi entre mot et phrase, explique sans doute la dualité stricte des pratiques génétiques de sa prose d'idées en général et de L'Idiot de la famille en particulier : d'un côté une simple prise de notes pour soi, de l'autre une rédaction continue pour autrui, sans brouillonage ou passage par un stade prérédactionnel, étapes intermédiaires non pertinentes pour la prose d'idées. La réconciliation des contraires (la possibilité de transformer de la signification en communication) est en effet, selon lui, le privilège, voire la fin constitutive, de la seule littérature : «l'écrivain écrivant, le vrai, je veux dire celui qui a les deux dimensions - en tout cas c'est comme ça pour moi - devrait faire de cette contradiction la matière même de son travail ${ }^{21}$.»

20. Nous renvoyons à nouveau à l'article mentionné ci-dessus.

2 I. «L'écrivain et son langage », art. cité, p. 49. 